\title{
Computational modelling of elastomeric materials to fit experimental data
}

\author{
S. Hernandez ${ }^{1}$, E. Menga ${ }^{2}$ \& S. Moledo ${ }^{1}$ \\ ${ }^{1}$ School of Civil Engineering, University of Coruña, Spain \\ ${ }^{2}$ Component Loads and Aeroelastic Department, EGLRG, \\ AIRBUS Operations, Spain
}

\begin{abstract}
The mitigation of the vibrations of components and structures, through the use of rubber mounts, is a common practice in many industries, such as in automotives and aeronautics or energy. It is a very important issue, because it is a key factor not just for the fatigue life but also for matters of comfort. On the other side, these industries make extensive use of finite element models to predict the dynamic behaviour of structures. Therefore, it means that the non-linear constitutive equations of rubber mount devices need to be properly integrated into the global analytical model. The quasi-static and dynamic behaviour of these devices can be quite complex, because they are usually done by a steel cover with an elastomer inside. Experimental test campaigns are usually carried forward to characterize the quasi-static and dynamic behaviour in terms of dynamic stiffness and loss factor. The experiments are designed to determine dependency on the frequency, the dynamic amplitude, the temperature and the preload.

In this paper an optimization methodology, combining hyper-elasticity, viscoelasticity and elasto-plasticity constitutive equations will be presented to obtain representative elastomeric behaviour, able to fit the experimental data in hand and to predict the rubber mount behaviour in load conditions different from those tested. The numerical results obtained are in very good agreement with the experimental data.

Keywords: elastomers, hyperelasticity, elastoplasticity, viscoelasticity, aircraft structures, optimization methods.
\end{abstract}




\section{Introduction}

Elastomers are a class of material present in many locations in aircraft structures. They are used as supporting devices, vibrations absorbers or joint elements. Nowadays, structural analysis of aircraft structures is done using finite element models and they must include all materials present in the aircraft. While metallic materials or even composites can be defined quite accurately by linear elastic behaviour, elastomers exhibit a more complex response. Experimental results show that hysteretic loops appear after the material is loaded and unloaded several times. The geometry of the loop depends on the material properties and the characteristic of the load. For giving material loads with constant frequency and varying amplitude produce a loop (as shown in figure 1(a)). On the other hand, a load with a constant amplitude and varying frequency corresponds to the loop represented in figure $1(\mathrm{~b})$.

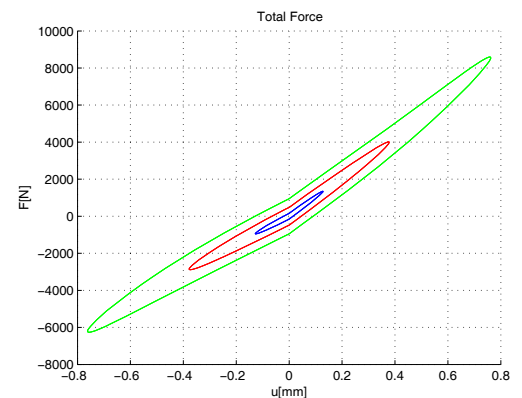

(a)

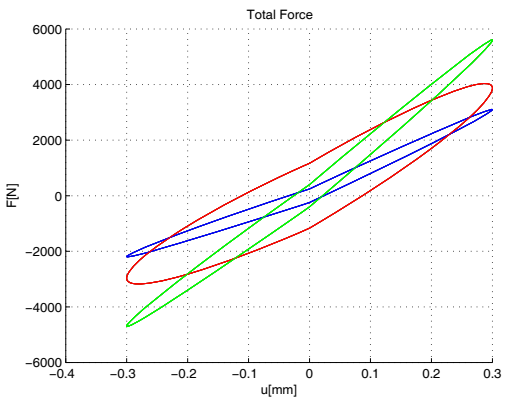

(b)

Figure 1: Shape of hysteretic loop: (a) constant frequency; (b) constant amplitude.

\section{Formulation of hyperelastic, viscoelastic and elastoplastic (HEVEP) element}

A quite comprehensive and efficient approach of defining elastomeric materials response is to consider them as a combination of hyperelastic, viscoelastic and elastoplastic behaviour (HEVEP). Consequently a description of the constitutive equations of these classes of materials is next presented.

\subsection{Formulation of hyperelastic element}

There are several formulations for hyperelastic elements depending, amongst other considerations, on their compressibility or incompressibility and relevant contributions have been made by Bonet and Wood [1], Shabana [2, 3], Belytschko et al. [4] or Martins et al. [5]. In our study, a model proposed by Yeoh [6] that has proven to be very efficient for rubber materials was selected. 
For this model the strain energy function $W$ is defined as a function of the first invariant of the right Cauchy-Green deformation tensor $I_{l}$.

$$
W\left(I_{1}\right)=\sum_{i=1}^{N} c_{i}\left(I_{1}-3\right)^{i}
$$

Usually $N=3$ and in some cases different coefficients are used for positive and negative deformations. In that case expression (1) becomes

$$
W\left(I_{1}\right)=\sum_{i=1}^{N} c_{i T}\left(I_{1}-3\right)^{i}+\sum_{i=1}^{N} c_{i C}\left(I_{1}-3\right)^{i}
$$

where $c_{i T}$ and $c_{i C}$ are the coefficients for tensile and compressive stresses. It is well known that invariants are expressed in term of the stretches $\lambda_{i}(i=1, \ldots 3)$

$$
I_{1}=\sum_{i=1}^{3} \lambda_{i}^{2} \quad I_{3}=\prod_{i=1}^{3} \lambda_{i}
$$

For incompressible materials $I_{2}=1$ and if the elastomeric material is subject to an uniaxial test it turns out

$$
\begin{gathered}
\lambda_{1}=\lambda \quad \lambda_{2}=\lambda_{3}=\frac{1}{\sqrt{\lambda}} \\
I_{1}=\lambda^{2}+\frac{2}{\lambda}
\end{gathered}
$$

Stress/strain relationship becomes according to Holzapfel [7].

$$
\sigma=2\left(\lambda^{2}-\frac{1}{\lambda}\right) \frac{\partial W}{\partial I_{1}}
$$

Thus, force/displacement relationship can be obtained by knowing that

$$
F_{H}=\sigma A_{o} \quad \lambda=\frac{l}{l_{o}}=\frac{l_{o}+u}{l_{o}}
$$

where $F_{H}$ is the applied force, $A_{o}$ specimen cross section, $l_{o}$ initial specimen length and $u$ the measured displacement in the uniaxial test.

\subsection{Formulation of elastoplastic material}

Elastoplastic behaviour is represented by a friction element. Force $F_{p}$ corresponding to the elastoplastic behaviour is established using the values of displacement $u_{p}$ that defines the sliding phase. In other words

$$
\begin{array}{lll}
\text { if } & u \leq u_{p} & F_{p}=k_{p} u \\
\text { if } & u>u_{p} & F_{p}=k_{p} u_{p}=F_{p \max }
\end{array}
$$




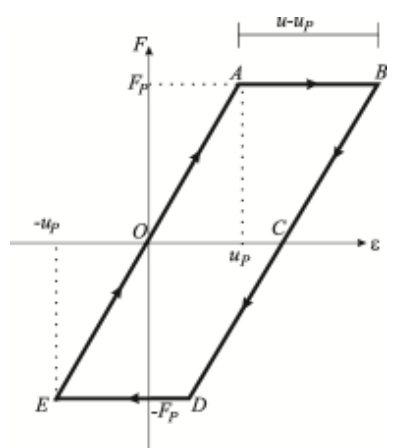

(a)

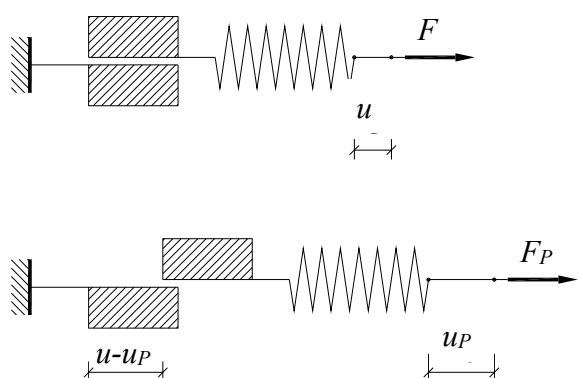

(b)

Figure 2: Behaviour of elastoplastic material: (a) hysteresis loop and (b) elastoplastic material model.

\subsection{Formulation of viscoelastic material}

Viscoelastic behaviour is modelled by using a Maxwell model composed of a dashpot and a linear spring that are defined by the relaxation time parameter $\tau$ and spring stiffness $k_{v}$.

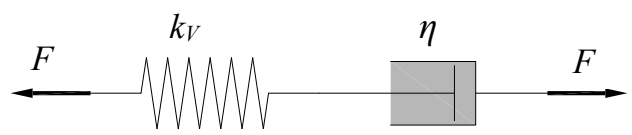

Figure 3: Components of the viscoelastic parameter.

Relaxation time parameter $\tau$ is defined as $\tau=\frac{\eta}{k_{V}}$ and the strain/displacement relationship at each load and unload step is:

a) Initial load branch

$$
\begin{gathered}
F_{V, l}=0 \\
F_{V, k+1}=F_{V, k} e^{-\frac{\Delta t}{\tau}}+\frac{k_{V} \Delta u}{2}\left(1+e^{-\frac{\Delta t}{\tau}}\right)
\end{gathered}
$$

b) Unload branch

$$
F_{V, k+1}=F_{V} e^{-\frac{\Delta t}{\tau}}+\frac{k_{V} \Delta u}{2}\left(1+e^{-\frac{\Delta t}{\tau}}\right)
$$

where the first value of $F_{v, k}$ is obtained at $u=u_{\max }$ 
c) Reload branch

$$
F_{V, k+1}=F_{V} e^{-\frac{\Delta t}{\tau}}+\frac{k_{V} \Delta u}{2}\left(1+e^{-\frac{\Delta t}{\tau}}\right)
$$

where the first value of $F_{v, k}$ is obtained at $u=u_{\text {min }}$

The complete cycle of initial load, unload and reload will produce a graph as shown in figure 4(a). After carrying out a new reload phase the final force is different from the one in the previous cycle as presented in figure 4(b). The same situation occurs in the forthcoming cycles. This behavior keeps on but in a attenuating way and finally, usually after about ten cycles although this number is problem dependent, a closed loop can be assumed as shown in figure 4(c).

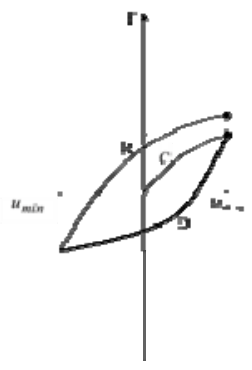

(a)

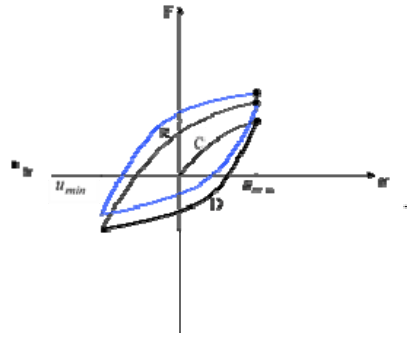

(b)

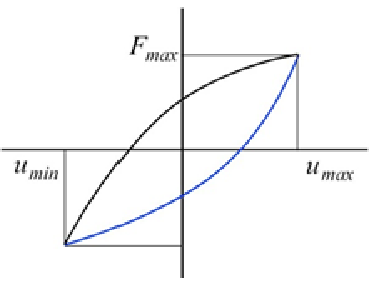

(c)

Figure 4: Evolution of the $F(u)$ curve: (a) initial cycle; (b) second unload cycle; (c) final hysteresis loop.

\section{Application example}

The formulation presented was applied to a set of experimental date of a class of elastomeric material used in aircraft structures. The objective of the study was to identify a proper combination of different constitutive equations that could set the hysteretic load shape. Two different strategies were carried out:

- Combination of hyperelastic and elastoplastic materials.

- Combination of hyperelastic, elastoplastic and viscoelastic materials.

In both cases the problem was solved by formulating an optimization problem defining an objective function $F$ which was an error metric consisting in the squares of the differences between values of the experimental curve $F_{E}\left(u_{i}\right)$ and the numerical one $F_{N}\left(u_{i}\right)$. Namely

$$
F=\sum_{i=1}^{N}\left(F_{E}\left(u_{i}\right)-F_{N}\left(u_{i}\right)\right)^{2}
$$

being $N$ the number of points considered on the hysteresis loop. 


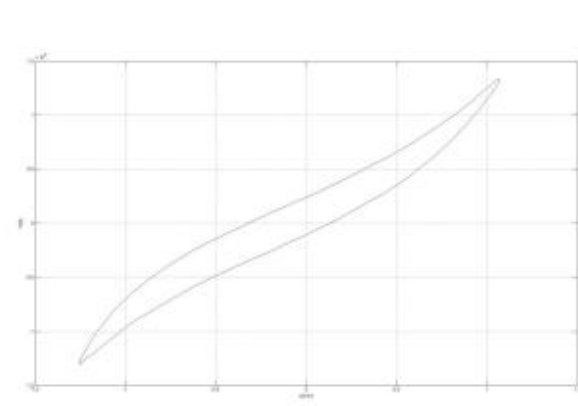

(a)

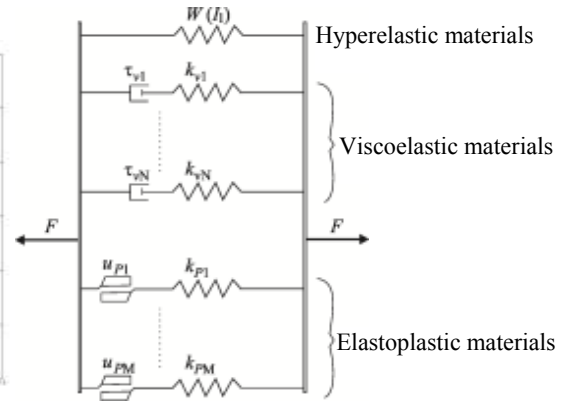

(b)

Figure 5: Hysteretic loop and materials considered in the study: (a) hysteretic loop; (b) scheme of material used.

Design values were composed by the coefficients of the Yeoh model, the value of the sliding displacement $u_{p}$ and stiffness $k_{p}$ of the elastoplastic elements and the stiffness $k_{V}$ and the relaxation time parameter $\tau$ of viscoelastic material.

\subsection{Fitting with hyperelastic and elastoplastic elements}

In this approach a combination of a hyperelastic element and three or six elastoplastic element were worked out. The idea of using different number of elastoplastic materials was to observe if this variation influenced the accuracy of the results at the end of the optimization problem. Different coefficients for compressive and tensile forces were used in the Yeoh model, so for the case of three elastoplastic materials the number of design variables was 12 and this figure increased up to 18 for the case with six elastoplastic materials. For the former case the numerical results obtained appear in Tables 1 and 2.

Table 1: Coefficients of the Yeoh model.

\begin{tabular}{|c|c|c|c|}
\cline { 2 - 4 } \multicolumn{1}{c|}{} & 1 & 2 & 3 \\
\hline $\mathrm{C}_{\mathrm{YT}}$ & 250.212675 & 145234.241 & 75699.3924 \\
\hline $\mathrm{C}_{\mathrm{YC}}$ & -414.679047 & 35389.3483 & 347426.707 \\
\hline
\end{tabular}

Table 2: Coefficients of the elastoplastic materials.

\begin{tabular}{|c|c|c|c|}
\cline { 2 - 4 } \multicolumn{1}{c|}{} & 1 & 2 & 3 \\
\hline $\mathrm{u}_{\mathrm{p}}$ & 0.02624775 & 0.84241707 & 1.05597988 \\
\hline $\mathrm{k}_{\mathrm{p}}$ & 12625.7817 & 2678.44495 & 4919.28876 \\
\hline
\end{tabular}

Figure 6 shows in red and green colour the contributions of hyperelastic and elastoplastic elements. By adding both responses a hysteretic loop that fits perfectly the experimental data is obtained. 


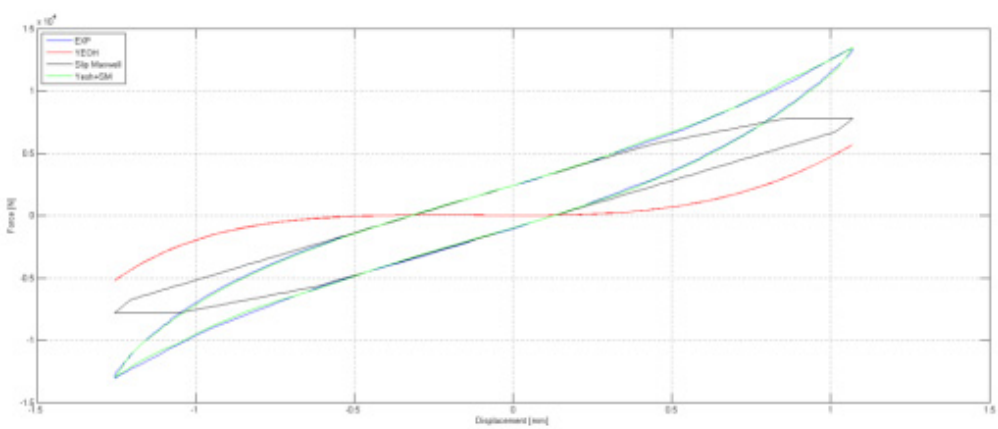

Figure 6: Fitting with a hyperelastic element and three elastoplastic elements.

When up to six elastoplastic elements were included the numerical values of the design variable at the end of the problem were the following.

Table 3: Coefficients of the Yeoh model.

\begin{tabular}{|c|c|c|c|}
\cline { 2 - 4 } \multicolumn{1}{c|}{} & 1 & 2 & 3 \\
\hline $\mathrm{C}_{\mathrm{YT}}$ & 1290.92693 & 127450.542 & 320694.448 \\
\hline $\mathrm{C}_{\mathrm{YC}}$ & 131.56443 & 33472.7572 & 354241.481 \\
\hline
\end{tabular}

Table 4: Coefficient of the elastoplastic materials.

\begin{tabular}{|c|c|c|c|c|c|c}
\cline { 2 - 7 } \multicolumn{1}{c|}{} & 1 & 2 & 3 & 4 & 5 & 6 \\
\hline $\mathrm{u}_{\mathrm{p}}$ & 0.00088092 & 0.74238013 & 1.06862969 & 0.05784354 & 0.92986345 & 0.95679066 \\
\hline $\mathrm{k}_{\mathrm{p}}$ & 219802.129 & 1139.9616 & 3752.92474 & 3250.38887 & 1236.36409 & 1148.69971 \\
\hline
\end{tabular}

Similarly to the previous formulation the individual contributions of each class of material and the combined values are presented in Figure 7 and again the experimental data are very much fitted.

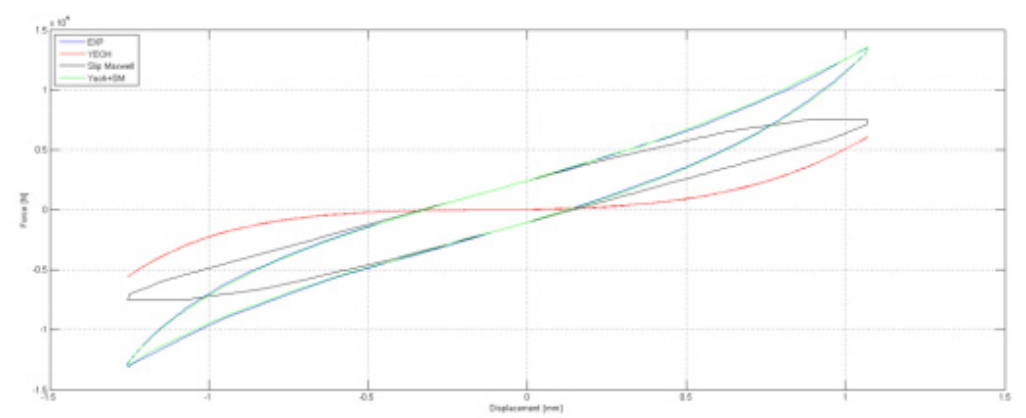

Figure 7: Fitting with a hyperelastic element and six elastoplastic materials. 


\subsection{Study considering hyperelastic, elastoplastic and viscoelastic elements}

As mentioned before, another study considering also viscoelastic material and thus, taking into account up to three different constitutive equations was carried out. Namely, the configurations analyzed were

- A hyperelastic element, a viscoelastic element and three elastoplastic elements.

- A hyperelastic element, a viscoelastic element and six elastoplastic elements.

Therefore, the first case led to an optimization problem with 14 design variables while the number of design variables was 20 in the second one. The numerical values obtained in the study with three elastoplastic variables are presented in the following tables.

Table 5: Coefficients of the Yeoh model.

\begin{tabular}{|c|c|c|c|}
\cline { 2 - 4 } \multicolumn{1}{c|}{} & 1 & 2 & 3 \\
\hline $\mathrm{C}_{\mathrm{YT}}$ & 14611.6962 & 92915.7921 & -656524.346 \\
\hline $\mathrm{C}_{\mathrm{YC}}$ & 9437.40184 & 17141.8514 & 54277.3558 \\
\hline
\end{tabular}

Table 6: Coefficients of elastoplastic materials.

\begin{tabular}{|c|c|c|c|}
\cline { 2 - 4 } \multicolumn{1}{c|}{} & 1 & 2 & 3 \\
\hline $\mathrm{u}_{\mathrm{P}}$ & 0 & 1.06862969 & 0.22986546 \\
\hline $\mathrm{k}_{\mathrm{P}}$ & 488.76247 & 805.581576 & 1331.85889 \\
\hline
\end{tabular}

The values of the parameters of the viscoelastic material turn out

$$
\tau=0.1522784 \quad k_{V}=7417.8974
$$

In Figure 8 the contribution of each class of material is presented using the same colours as in the previous paragraph. It can be observed that the experimental data are very accurately reproduced.

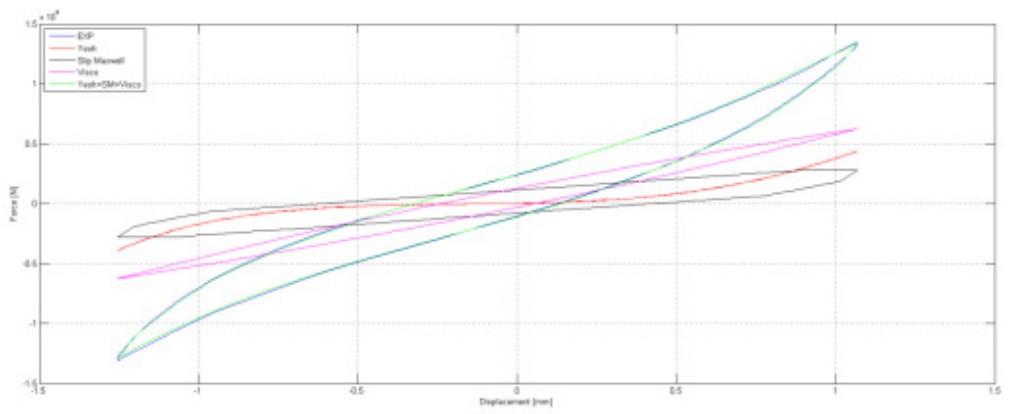

Figure 8: Fitting using a hyperelastic and viscoelastic element and 3 elastoplastic elements. 
Finally, a study increasing up to six elastoplastic elements was carried out. The numerical values for the viscoelastic material were

$$
\tau=0.11954619 \quad k_{V}=6452.33768
$$

Values of coefficients of the remaining types of materials are presented in the following tables.

Table 7: $\quad$ Coefficients of the Yeoh model.

\begin{tabular}{|l|c|c|c|}
\cline { 2 - 4 } \multicolumn{1}{c|}{} & 1 & 2 & 3 \\
\hline $\mathrm{C}_{\mathrm{YT}}$ & 14610.9185 & 92915.7921 & -656524.346 \\
\hline $\mathrm{C}_{\mathrm{YC}}$ & 9437.40184 & 17141.8514 & 54277.3558 \\
\hline
\end{tabular}

Table 8: Coefficients of elastoplastic materials.

\begin{tabular}{|c|c|c|c|c|c|c|}
\cline { 2 - 7 } \multicolumn{1}{c|}{} & 1 & 2 & 3 & 4 & 5 & 6 \\
\hline $\mathrm{k}_{\mathrm{P}}$ & 0.20753566 & 0 & 0.26664412 & 1.06862969 & 0.14596869 & 0.30561166 \\
\hline $\mathrm{k}_{\mathrm{P}}$ & 389.959727 & 718.412669 & 703.194874 & 821.455335 & 1568.759 & 687.826133 \\
\hline
\end{tabular}

In figure 9 the individual contributions and the combined values are presented showing a perfect matching of the experimental data.

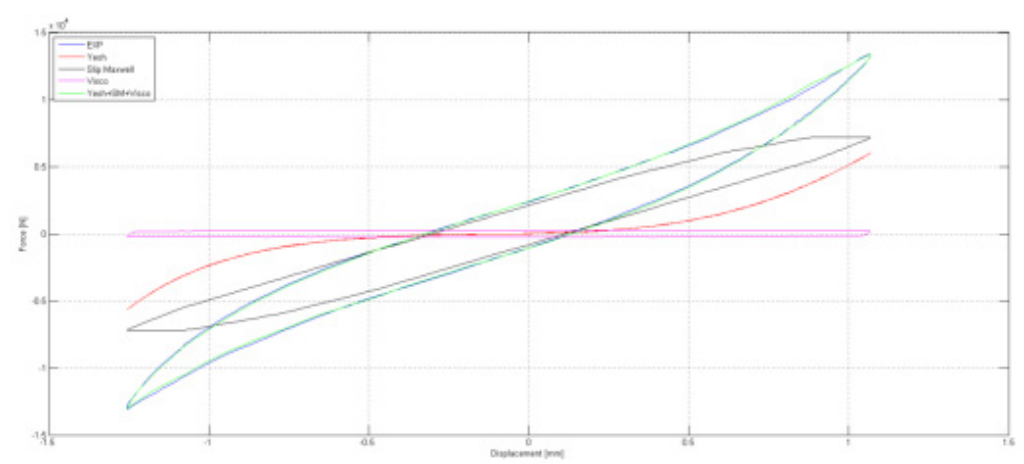

Figure 9: Fitting with a hyperelastic and viscoelastic element and 6 elastoplastic elements.

\section{Conclusions}

The following expressions can be drawn from this work:

1) Elastomers are a crucial component in aircraft structures and proper identification of their mechanical properties is very much needed for accurate modeling in finite element structural models.

2) Efficient modeling of elastomeric materials requires a combination of several nonlinear behaviour as hyperelasticity, elastoplasticity and viscoelasticity. 
3) A formulation for combining such constitutive equations in order to model the type of elastomers used in aircraft industry has been worked out.

4) An application example using data very similar to experiments of real material has been used to prove the capability of the method and the numerical values led to a perfect fitting of the shape of the hysteretic loop considered.

\section{References}

[1] Bonet, J. and Wood, R.D., Nonlinear Continuum Mechanics for Finite Element Analysis, Cambridge University Press, 1997.

[2] Shabana A. A, Dynamic of Multibody Systems, 3rd Edition, Cambridge University Press, 2005.

[3] Shabana A. A, Computational Continuum Mechanics, Cambridge University Press, 2008.

[4] Belytschko, T., Lin, W.K. and Moran, B., Non linear Finite Elements for Continua and Structures, John Wiley and Sons, New York, 2000.

[5] Martins, P.A.L.S., Natal Jorge, R. M. and Ferrera, J. M., A Comparative Study of Several Material Models for Prediction of Hyperelastic Properties: Application to Silicone-Rubber and Soft Tissues, Strain, Vol. 42, Issue 3, pp. 135-147, 2006.

[6] Yeoh, O. H., Some forms of the strain energy function for rubber, Rubber Chemistry and Technology, Vol. 66, Issue 5, pp 754-771, 1993.

[7] Hotzapfel, G. A., Nonlinear Solid Mechanics: A Continuum Approach for Engineering, John Wiley and Sons, 2000. 